\title{
Machine Learning-Based Prediction of Distribution Network Voltage and Sensor Allocation
}

\author{
Alvaro Furlani Bastos ${ }^{1}$ (presenter), Surya Santoso ${ }^{1}$, Venkat Krishnan², Yingchen Zhang \\ ${ }^{1}$ The University of Texas at Austin, ${ }^{2}$ National Renewable Energy Laboratory (NREL) \\ Corresponding author: alvaro.fbastos@utexas.edu \\ Best Conference Papers on Distribution Systems, Energy Hubs, and Smart Grids \\ Monday, August 3, 2020
}

This work was authored in part by the National Renewable Energy Laboratory (NREL), operated by Alliance for Sustainable Energy, LLC, for the U.S. Department of Energy (DOE) under Contract No. DE-AC36-08GO28308. Funding provided by the U.S. Department of Energy Office of Electricity Delivery and Energy Reliability (DEOE0000876). The views expressed in the article do not necessarily represent the views of the DOE or the U.S. Government. The U.S. Government and the publisher, by accepting the article for publication, acknowledges that the U.S. Government retains a nonexclusive, paid-up, irrevocable, worldwide license to publish or reproduce the published form of this work, or allow others to do so, for U.S. Government purposes. 


\section{Motivation}

\section{Background:}

- Large photovoltaic integration at the distribution level challenges the traditional voltage control techniques.

- Distribution networks are usually not fully monitored and state estimation is less developed in distribution systems than in transmission systems.

- Large-scale deployment of smart meters increases visibility into distribution networks.

\section{Objectives:}

- Predict bus voltages through historical data and load/generation forecast.

$\checkmark$ Learn a mapping function between load sizes and bus voltages without full knowledge of the circuit topology.

$\checkmark$ Identify strategic buses for monitoring to achieve high prediction accuracy. 


\section{Voltage Prediction: Single Regressors}

Regressor MAE $\left(10^{-3} \mathrm{pu}\right)$ RMSE $\left(10^{-3} \mathrm{pu}\right)$ Max. error $\left(10^{-3} \mathrm{pu}\right) \quad \%$ Best

MAE: mean absolute error

RMSE: root mean square error

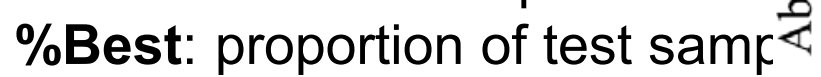

\begin{tabular}{|lllll|}
\hline Bayesian ridge & 1.563 & 1.986 & 10.66 & 7.22 \\
\hline Linear regression & 1.563 & 1.986 & 10.65 & 8.78 \\
\hline Lasso-Lars & 1.661 & 2.161 & 13.53 & 22.44 \\
\hline Gradient boosting & 1.788 & 2.246 & 11.43 & 26.94 \\
\hline
\end{tabular}

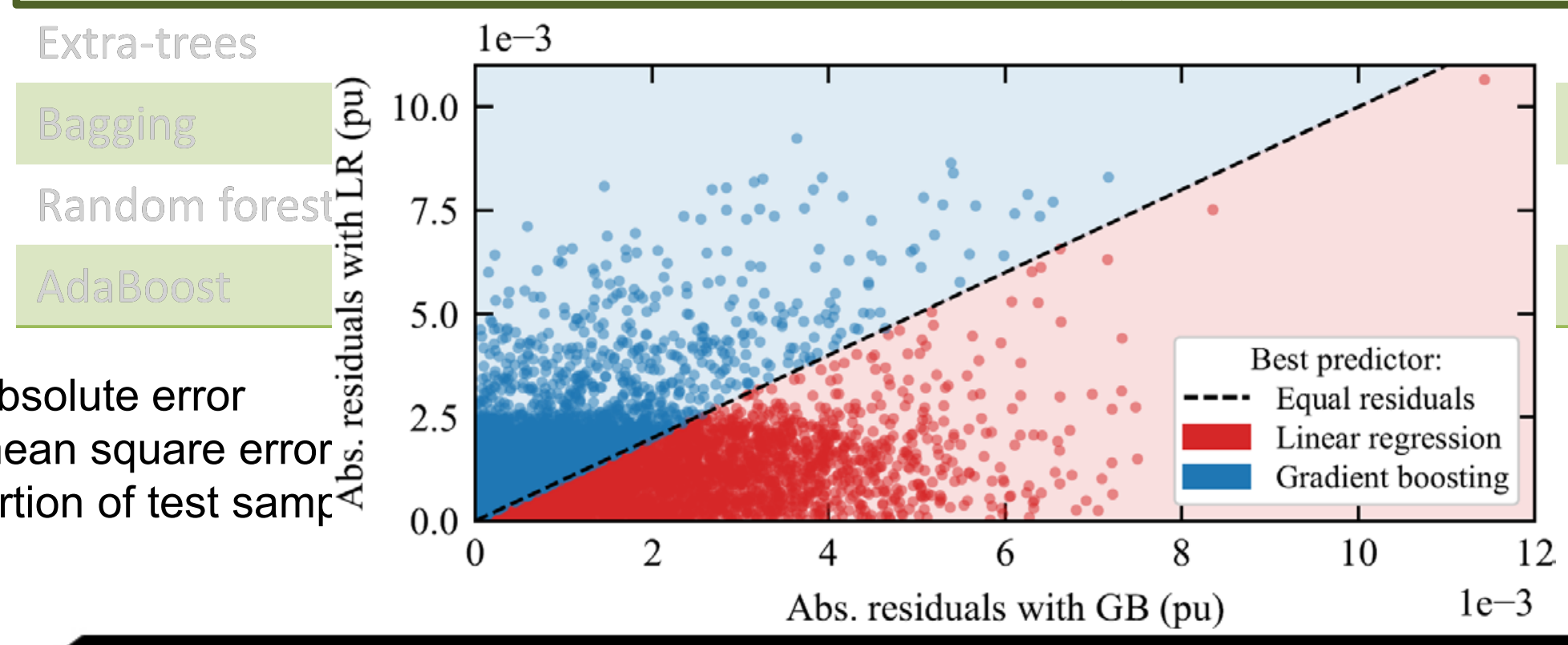

\section{Ensemble regressor:}

- MAE decreased by $26 \%$.

- Maximum error still larger than 0.01 p.u.

$$
1 \mathrm{e}-3
$$




\section{Voltage Prediction: Two-Step Regressor}

- Proposed two-step regressor:

$\checkmark$ Split the range of target values into three subranges: less than $(1-\tau)$ pu (submodel $\mathbf{A}$ ), between $(1-\tau)$ and $(1+\tau)$ pu (submodel B), and greater than $(1+\tau)$ pu (submodel $\mathbf{C}$ ).

$\checkmark$ Fit a separate model for each subrange.

- Two-step voltage prediction:

$\checkmark$ Predict voltage using a global regressor (single model).

Two-step regressor:

- MAE decreased by $69 \%$.

- Maximum error decreased to 0.0034 p.u.

$\checkmark$ Based on the initial prediction, select the corresponding local regressor (submodel A, B, or C) and perform the voltage prediction again (refinement). 


\section{Sensor Allocation}

- Trade-off between fewer measurements at strategic locations and network observability.

- Similar buses are identified through agglomerative hierarchical clustering.

- Proposed approach:

$\checkmark$ Select the most important bus from each cluster for monitoring.

$\checkmark$ Add more buses (in descending order of importance) to the predictor until the desired accuracy is achieved or there is no further improvement in the prediction error.
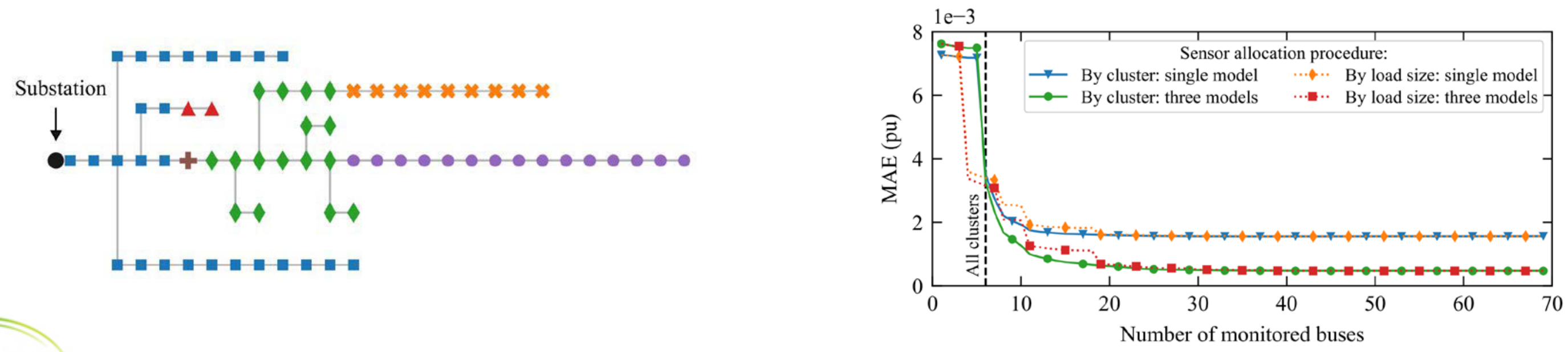


\section{Conclusions/Recommendations}

- Accuracy of voltage prediction in distribution networks can be significantly improved by modifying and combining simple estimation techniques.

- High network observability can be achieved by selecting only a few strategic buses for monitoring.

- This data-driven estimation approach can be updated periodically to reflect changes in the network. 